\title{
Ecuador Forest Plots Database
}

\author{
Jürgen Homeier
}

\begin{abstract}
The Ecuador Forest Plots Database (GIVD ID SA-EC-001) contains permanent plot data (tree species composition and environmental data) from different ecoregions in Ecuador (i.e. from tropical lowland to upper montane forests, tropical dry forest). The permanent plots have been established to study forest structure and tree species composition along environmental gradients.
\end{abstract}

Keywords: forest type; tree species composition.

\begin{tabular}{|c|c|c|}
\hline \multirow{2}{*}{\multicolumn{3}{|c|}{$\begin{array}{l}\text { GIVD Database ID: SA-EC-001 } \\
\text { Ecuador Forest Plots Database }\end{array}$}} \\
\hline & & \\
\hline \multicolumn{3}{|c|}{$\begin{array}{l}\text { Scope: The database contains permanent plot data (tree species composition and environmental data) from different ecoregions in Ecuador (i.e. } \\
\text { from tropical lowland to upper montane forests, tropical dry forest) from S and NE Ecuador. }\end{array}$} \\
\hline Status: ongoing capture & \multicolumn{2}{|c|}{ Period: $2000-2010$} \\
\hline \multicolumn{3}{|c|}{ Database manager(s): Jürgen Homeier (jhomeie@gwdg.de) } \\
\hline \multicolumn{3}{|c|}{ Owner: Juergen Homeier } \\
\hline \multicolumn{3}{|l|}{ Web address: $[\mathrm{NA}]$} \\
\hline Availability: according to a spe & Online upload: no & Online search: no \\
\hline Database format(s): 4th Dimen & \multicolumn{2}{|c|}{ Export format(s): Excel, CSV file, plain text file } \\
\hline \multicolumn{3}{|l|}{ Publication: [NA] } \\
\hline Plot type(s): normal plots & \multicolumn{2}{|c|}{ Plot-size range: $400-400 \mathrm{~m}^{2}$} \\
\hline Non-overlapping plots: 230 & Estimate of existing plots: $[N A]$ & Completeness: $[N A]$ \\
\hline Total plot observations: 230 & Number of sources: $[\mathrm{NA}]$ & Valid taxa: 1,000 \\
\hline \multicolumn{3}{|l|}{ Countries: EC: $100.0 \%$} \\
\hline \multicolumn{3}{|c|}{ Forest: $100 \%$ — Non-forest: aquatic: 0\%; semi-aquatic: $0 \%$; arctic-alpine: $0 \%$; natural: $0 \%$; semi-natural: $0 \%$; anthropogenic: $0 \%$} \\
\hline \multicolumn{3}{|c|}{ Guilds: all vascular plants: $10 \%$; only trees and shrubs: $90 \%$} \\
\hline \multicolumn{3}{|c|}{ Environmental data: altitude: $100 \%$; slope aspect: $95 \%$; slope inclination: $95 \%$; soil pH: $95 \%$} \\
\hline \multicolumn{3}{|c|}{ Performance measure(s): number of individuals: $100 \%$; measurements like diameter or height of trees: $100 \%$; biomass: $50 \%$} \\
\hline \multicolumn{3}{|c|}{ Geographic localisation: GPS coordinates (precision $25 \mathrm{~m}$ or less): $95 \%$; point coordinates less precise than GPS, up to $1 \mathrm{~km}: 5 \%$} \\
\hline \multicolumn{3}{|c|}{ Sampling periods: $2000-2009: 90.0 \% ; 2010-2019: 10.0 \%$} \\
\hline Information as of & Irther details and future updates available & \\
\hline
\end{tabular}

Jürgen Homeier (jhomeie@gwdg.de)

Department of Plant Ecology and Ecosystems Research, University of Göttingen, Untere Karspüle 2, 37073 Göttingen, GERMANY 